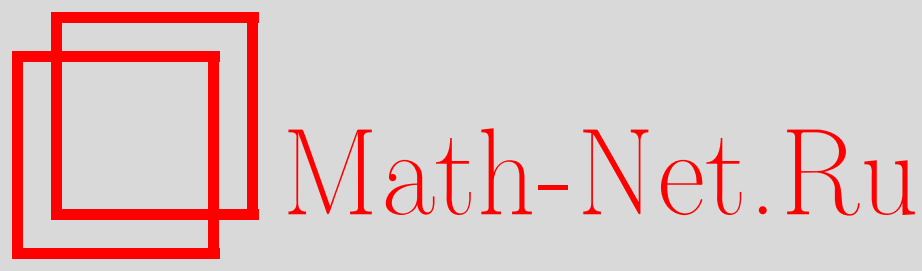

В. Н. Лихачев, Т. Ю. Астахова, Г. А. Виноградов, “Электронный пинг-понг" на одномерной решетке. Многократные отражения волнового пакета и захват волновой функции акцептором, ТМФ, 2013, том 176, номер 2, 306321

DOI: https://doi.org/10.4213/tmf8435

Использование Общероссийского математического портала Math-Net.Ru подразумевает, что вы прочитали и согласны с пользовательским соглашением http://www . mathnet.ru/rus/agreement

Параметры загрузки:

IP : 34.227 .88 .159

26 апреля 2023 г., $17: 34: 48$

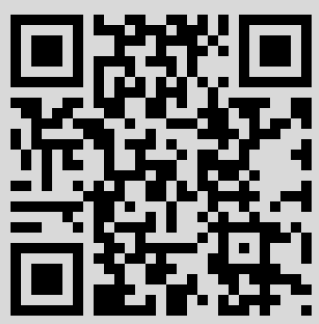


Том 176, № 2

август, 2013

(C) 2013 г. В.Н. Лихачев*, Т. Ю. Астахова*, Г. А. Виноградов*

\section{“ЭЛЕКТРОННЫЙ ПИНГ-ПОНГ” НА ОДНОМЕРНОЙ РЕШЕТКЕ. МНОГОКРАТНЫЕ ОТРАЖЕНИЯ ВОЛНОВОГО ПАКЕТА И ЗАХВАТ
ВОЛНОВОЙ ФУНКЦИИ АКЦЕПТОРОМ}

Рассмотрена задача о многократных отражениях волновой функции от концов решетки, при этом обнаружено интересное явление концентрации амплитуды волновой функции на примесном центре после отражений. Решение, полученное разложением полной волновой функции на примесном узле $a(t)$ по парциальным амплитудам $a_{k}(t)$, вклад каждой из которых становится существенным только после $k$-го отражения от конца решетки, очень хорошо согласуется с результатами численного моделирования. Решена проблема захвата волновой функции акцептором. Полученные результаты могут помочь в объяснении экспериментальных данных по переносу заряда в искусственных олигонуклеотидах и полипептидах. В некоторых предельных случаях найдены выражения для вероятности захвата электрона, что может служить оценкой для квантового выхода переноса заряда.

Ключевые слова: перенос заряда, волновой пакет.

DOI: $10.4213 / \operatorname{tmf} 8435$

\section{1. ПОСТАНОВКА ЗАДАЧИ}

Настоящая работа является продолжением нашей статьи [1], посвященной задаче об эволюции первоначально локализованной волновой функции в решеточной модели. Для полноты изложения повторим некоторые основные положения, касающиеся задачи об отражении и возвращении возбуждения к началу решетки, однако изложение будет немного отличаться от использованного в работе [1]. Итак, мы рассматриваем квантовую систему, задаваемую $(N+1) \times(N+1)$-матрицей. Система состоит из выделенного крайнего левого узла с энергией $E$ и резервуара, задаваемого матрицей $\widehat{\mathbf{B}}$ размерности $N \times N$. Гамильтониан записывается в виде

$$
H=\left(\begin{array}{ll}
E & \overrightarrow{\mathbf{v}} \\
\overrightarrow{\mathbf{v}} & \widehat{\mathbf{B}}
\end{array}\right)
$$

* Институт биохимической физики им. Н. М. Эмануэля РАН, Москва, Россия. E-mail: gvin@deom.chph.ras.ru 
с волновой функцией $\vec{\Psi}(t)=\{a(t), \vec{b}(t)\}=a(t), b_{1}(t), b_{2}(t), \ldots, b_{N}(t)$. Взаимодействие выделенного узла и резервуара задается вектором $\overrightarrow{\mathbf{v}}$. Матрица $\widehat{\mathbf{B}}-$ трехдиагональная матрица с нулевыми значениями на главной диагонали и единичными значениями на обеих побочных диагоналях. Начальное условие имеет вид $a(t=0)=1$, $\overrightarrow{\mathbf{b}}(t=0)=0$. Задачей является вычисление эволюции состояния волновой функции на примесном узле, т. е. нахождение зависимости амплитуды $a(t)$ от времени.

Уравнение Шредингера для рассматриваемой системы в безразмерном виде $(\hbar=1)$ выглядит следующим образом:

$$
\begin{aligned}
i \frac{d a(t)}{d t}=E a(t)+(\overrightarrow{\mathbf{v}} \cdot \overrightarrow{\mathbf{b}}), & a(t=0)=1, \\
i \frac{d \overrightarrow{\mathbf{b}}(t)}{d t}=(\widehat{\mathbf{B}} \cdot \overrightarrow{\mathbf{b}}(t))+\overrightarrow{\mathbf{v}} a(t), & \overrightarrow{\mathbf{b}}(t=0)=0 .
\end{aligned}
$$

Если разложить вектор $\overrightarrow{\mathbf{b}}$ по собственным функциям $b_{k}(i)$ матрицы $\widehat{\mathbf{B}}$, то для амплитуды $a(t)$ получается интегродифференциальное уравнение

$$
\dot{a}=-i E a-\int_{0}^{t} B^{N}(t-\tau) a(\tau) d \tau, \quad a(t=0)=1,
$$

где ядро определяется выражением

$$
B^{N}(t)=\sum_{k=1}^{N} e^{-i \varepsilon_{k} t}\left(\overrightarrow{\mathbf{v}} \cdot \overrightarrow{\mathbf{b}}_{k}\right)^{2}
$$

где $\varepsilon_{k}$ - энергия $k$-го собственного значения резервуара.

Резервуар $\widehat{\mathbf{B}}$ задается трехдиагональной матрицей с матричными элементами $B_{i, j}=\delta_{i, j+1}+\delta_{i, j-1}$. Это означает, что он представляет собой решетку из $N$ узлов, а возбуждение (электрон) может перескакивать только на ближайшие соседние узлы. Энергию электрона на узлах положим равной нулю, что соответствует выбору начала отсчета энергии. Интеграл перескока выберем равным единице, что соответствует выбору единицы энергии, равной интегралу перескока.

Взаимодействие $\overrightarrow{\mathbf{v}}$ примесного узла с решеткой (резервуаром) мы выберем в виде $v_{i}=C \delta_{i, 1}$, т. е. с примесного узла возбуждение может переходить только на первый узел решетки (резервуара) и обратно, и $C$ есть интеграл перескока (типичное значение интеграла перескока для ДНК $\sim 0.3$ эВ).

Уравнение Шредингера (2) для этой модели приобретает следующий вид:

$$
\begin{aligned}
i \dot{a} & =E a+C b_{1}, \\
i \dot{b}_{1} & =b_{2}+C a, \\
i \dot{b}_{2} & =b_{1}+b_{3}, \\
\ldots \ldots \ldots \ldots \ldots & \ldots \ldots \\
i \dot{b}_{N} & =b_{N-1}
\end{aligned}
$$

с начальными условиями $a(0)=1, b_{i=1,2, \ldots, N}(0)=0$, что соответствует нахождению электрона в начальный момент на примесном узле. Собственные значения $\varepsilon(k)$ 
и собственные функции $b_{i}(k)$ матрицы резервуара хорошо известны:

$$
\varepsilon(k)=2 \cos \left(\frac{\pi k}{N+1}\right), \quad b_{k}(i)=\sqrt{\frac{2}{N+1}} \sin \left(\frac{\pi k}{N+1} i\right), \quad k=1,2, \ldots, N .
$$

Тогда в соответствии с выражениями (6) ядро $B^{N}(t)$ уравнения (3) определяется суммой

$$
B^{N}(t)=\frac{2 C^{2}}{N+1} \sum_{k=1}^{N} \sin ^{2}\left(\frac{\pi k}{N+1}\right) e^{-2 i \cos (\pi k /(N+1)) t}
$$

\section{2. РАЗЛОЖКНИЕ $A(T)$ ПО ПАРЦИАЛЬНЫМ АМПЛИТУДАМ}

В работе [1] было показано, что на достаточно длинных решетках образуется хорошо сформированный импульс с крутым передним фронтом. Скорость фронта импульса $v=2$ есть максимально возможная групповая скорость. Таким образом, если решетка состоит из $N$ узлов, то время возвращения импульса обратно к примесному узлу равно $t \approx N$, а цикл из $k$ полных возвращений займет время $t \approx k N$. Там же было подробно исследовано поведение амплитуды $a(t)$ для времен $t \lesssim N$. Сейчас нашей задачей является нахождение амплитуды $a(t)$ для всех времен, и мы будем искать решение уравнения (3) с ядром, задаваемым выражением (7).

Чтобы построить решение уравнения (3) для амплитуды $a(t)$ и описать возвращение начального возбуждения на исходный примесный узел, удобно представить амплитуду $a(t)$ в виде суммы парциальных амплитуд $a_{k}(t)$, каждая из которых пренебрежимо мала на временах $t \lesssim k N$, а ее вклад в полную амплитуду $a(t)$ становится существенным начиная лишь с момента времени $t \sim k N$.

Чтобы увидеть возможность подобного представления, нужно преобразовать ядро $B^{N}(t)$ исходного уравнения (7) по формуле Пуассона:

$$
\begin{aligned}
B^{N}(t) & =C^{2} \sum_{m=-\infty}^{m=+\infty} B_{m}(t) \Rightarrow \\
\Rightarrow \quad B_{m}(t) & =\frac{2}{\pi} \int_{0}^{\pi} d x \sin ^{2} x e^{-2 i[m(N+1) x+t \cos x]} .
\end{aligned}
$$

Отметим, что $B_{m}(t)$ - вещественная функция, а для $B_{0}(t)$ есть явное представление через функции Бесселя:

$$
B_{0}(t)=J_{0}(2 t)+J_{2}(2 t) .
$$

С учетом преобразования (8) исходное уравнение (3) перепишется следующим обра3ом:

$$
\dot{a}(t)=-i E a(t)-C^{2} \sum_{m=-\infty}^{\infty} \int_{0}^{t} B_{m}\left(t-t^{\prime}\right) a\left(t^{\prime}\right) d t^{\prime} .
$$

Отметим важное и полезное для последующего анализа свойство функций $B_{m}$ : в области $t<m N$ функция $B_{m}(t)$ мала, а именно

$$
B_{m}(t) \approx \frac{\sin (2 t)}{\pi[m(N+1)]^{3}}, \quad m(N+1)-t \gg 1
$$


С достаточной точностью можно считать, что функция $B_{m}(t) \approx 0$, пока $t<m N$. Следовательно, можно полагать, что пока $t<m N$, то в основном уравнении (10) существенны только члены $B_{k}$ c $k<m$. Теперь представим амплитуду $a(t)$ в виде суммы парциальных амплитуд:

$$
a(t)=a_{0}(t)+a_{1}(t)+a_{2}(t)+\cdots .
$$

Напомним, что наше предположение относительно амплитуд $a_{k}(t)$ состоит в том, что амплитуда $a_{k}(t)$ мала при $t<k N$ и становится существенной лишь при $t \sim k N$. В каком-то смысле $a_{k}(t)$ ведет себя подобно $B_{k}(t)$. Строя уравнение для $a_{k}(t)$, будем считать, что в правую часть уравнения (10) будут входить лишь $B_{m}(t)$ с $m \leqslant k$.

Прежде чем написать уравнения для $a_{k}(t)$, сделаем одно замечание. Члены $B_{m}(t)$ малы при $m<0$ (даже для недлинных решеток). Эта малость обеспечивается тем, что $B_{-m}(t)=B_{m}(-t)$, следовательно, в рассматриваемой области $(t>0) B_{m}(t) \sim$ $(m N)^{-3}$. Тем не менее эти члены будут учитываться, так как оказывается, что это позволяет получить точные аналитические соотношения. Для учета $B_{m}(t), m<0$, введем функции

$$
\widetilde{B}_{m}(t)=B_{m}(t)+B_{-m}(t), \quad m>0,
$$

и для сохранения единства обозначений будем полагать, что $\widetilde{B}_{0}(t) \equiv B_{0}(t)$.

Теперь в соответствии с нашим предположением превратим одно уравнение (10) в систему зацепляющихся уравнений для парциальных амплитуд $a_{k}(t)$. Уравнение для $a_{0}(t)$ будет содержать в правой части только функцию $\widetilde{B}_{0}(t)$ (см. представление $(9))$ :

$$
\dot{a}_{0}=-i E a_{0}-C^{2} \int_{0}^{t} \widetilde{B}_{0}\left(t-t^{\prime}\right) a_{0}\left(t^{\prime}\right) d t^{\prime}, \quad a_{0}(0)=1 .
$$

В соответствии с вышеприведенными соображениями уравнение для $a_{k}(t), k>0$, запишем в виде

$$
\dot{a}_{k}=-i E a_{k}-C^{2} \int_{0}^{t} \sum_{m=0}^{k} \widetilde{B}_{m}\left(t-t^{\prime}\right) a_{k-m}\left(t^{\prime}\right) d t^{\prime}, \quad a_{k}(0)=0, \quad k>0 .
$$

Отметим, что система уравнений (15) является точной.

Преобразование Лапласа уравнения (14) для $a_{0}(t)$ дает следующее выражение для $a_{0}(p)$ :

$$
a_{0}(p)=\frac{1}{p+i E+C^{2} \widetilde{B}_{0}(p)},
$$

где $\widetilde{B}_{0}(p)$ - преобразование Лапласа функции $B_{0}(t)$ (см. представление $\left.(9)\right)$ :

$$
\widetilde{B}_{0}(p)=\frac{1}{2}\left(\sqrt{p^{2}+4}-p\right) .
$$

Напомним, что выражение (16) есть амплитуда $a(t)$ в бесконечной цепочке, когда нет возвратов.

В свою очередь, преобразование Лапласа системы уравнений (15) дает алгебраическую систему рекуррентных соотношений для $a_{k}(p), k>0$ :

$$
a_{k}(p)=-C^{2} a_{0}(p) \sum_{m=0}^{k-1} \widetilde{B}_{k-m}(p) a_{m}(p) .
$$




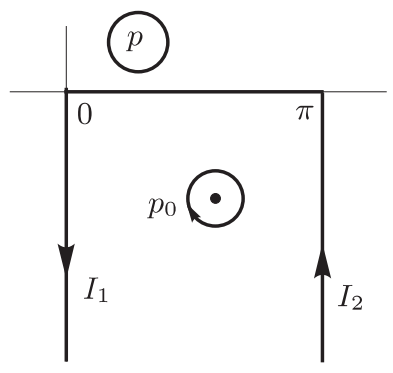

Рис. 1. Деформация контура интегрирования: интегрирование $[0, \pi]$ в выражении (19) заменяется вычетом в полюсе $p_{0}$ и двумя интегралами $I_{1}$ и $I_{2}$.

Преобразование Лапласа $\widetilde{B}_{m}(p)$ функции $\widetilde{B}_{m}(t)$ (см. формулу $\left.(8)\right)$ дается интегралом

$$
\widetilde{B}_{m}(p)=\frac{2}{\pi} \int_{0}^{t} \sin ^{2} y e^{-2 i m(N+1) y}\left[\frac{1}{p+2 i \cos y}+\frac{1}{p-2 i \cos y}\right] d y .
$$

Для интеграла (19) можно получить явное выражение. С этой целью деформируем контур интегрирования так, как показано на рис. 1. Подынтегральная функция имеет период $\pi$, и интегралы $I_{1}$ и $I_{2}$ сокращаются, так как проходятся в противоположных направлениях. Следовательно, $\widetilde{B}_{m}(p)$ определяется только полюсным слагаемым в точке $y_{0}, \cos y_{0}=i p / 2$. Тогда получаем

$$
\widetilde{B}_{m}(p)=\sqrt{4+p^{2}}\left[i \widetilde{B}_{0}(p)\right]^{2 m(N+1)} .
$$

Благодаря тому удачному обстоятельству, что $\widetilde{B}_{m}(p)$ образуют геометрическую прогрессию по $m$, можно провести суммирование в рекуррентных формулах (18). Именно это обстоятельство позволяет записать полную амплитуду $a(t)$ в виде замкнутого выражения, что и сделано в приложении. Для лапласовских образов парциальных амплитуд $a_{k}(p), k>0$, получаются следующие выражения:

$$
a_{k}(p)=-a_{0}(p) d(1-d)^{k-1}\left(i \widetilde{B}_{0}\right)^{2 k(N+1)} \quad \Rightarrow \quad d \equiv a_{0} C^{2} \sqrt{p^{2}+4} .
$$

Теперь для получения явных выражений для парциальных амплитуд делаем обратное преобразование Лапласа. Сворачивая лапласовский интеграл вокруг разреза $[-2 i, 2 i]$, для $a_{0}(t)$ получаем

$$
a_{0}(t)=\frac{1}{\pi} \int_{-2}^{2} e^{i x t} \operatorname{Im}\left\{\left[x\left(1-\frac{C^{2}}{2}\right)+E-\frac{i C^{2}}{2} \sqrt{4-x^{2}}\right]^{-1}\right\} d x .
$$

Далее приходим к следующему выражению для парциальных амплитуд $a_{k}(t)$ :

$$
a_{k}(t)=-\frac{C^{2}}{\pi} \int_{-2}^{2} \sqrt{4-x^{2}} e^{i x t} \operatorname{Re}\left[a_{0}^{2}\left(1-C^{2} a_{0} \sqrt{4-x^{2}}\right)^{k-1}\left(i \widetilde{B}_{0}\right)^{2 k(N+1)}\right] d x .
$$

Здесь преобразования Лапласа для $a_{0}(p)$ (см. формулы $\left.(16),(20)\right)$ берутся при $p=i x$. 


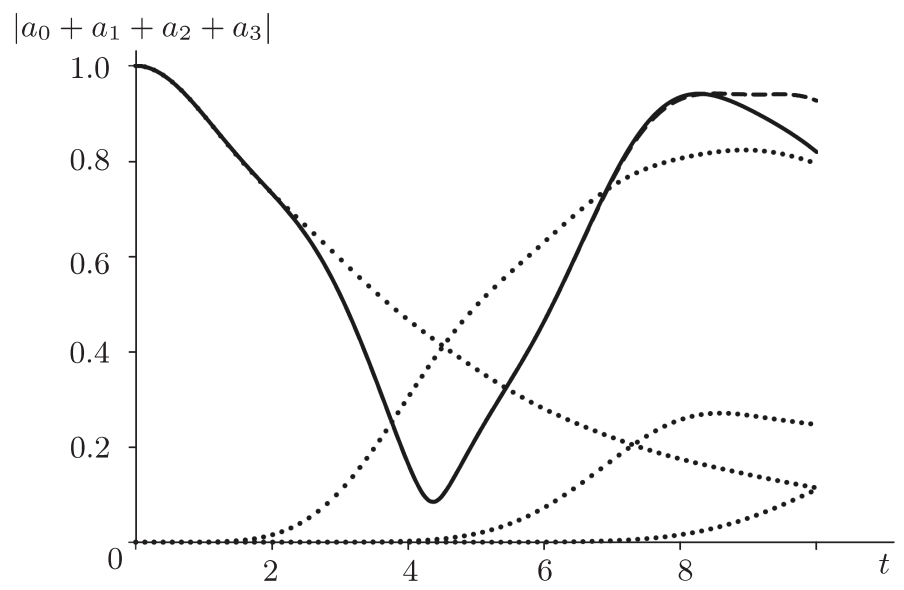

Рис. 2. Сравнение ограниченной суммы парциальных амплитуд $a_{0}+a_{1}+$ $a_{2}+a_{3}$ (сплошная линия) с численным интегрированием (штриховая линия). Пунктир - амплитуды $a_{0}, a_{1}, a_{2}$ и $a_{3}$, "начинающиеся" при $t=0,2,4,6$, соответственно. Малое расхождение наблюдается только при $t>8$ - там, где начинает "работать" неучтенная амплитуда $a_{4}$. Значения параметров: $N=2$, $E=1, C=0.5$. Среднеквадратичное отклонение $\lesssim 0.0014$.

Анализ выражения для $a_{k}(t)$ показывает, что, как и предполагалось, $a_{k}(t) \ll 1$ при $t<(k+1) N$. Следовательно, если для представления амплитуды $a(t)$ взять частичную сумму $a_{0}+a_{1}+\cdots+a_{k}$, то погрешность такого приближения имеет тот же порядок, что и $\widetilde{B}_{k}(t)$ (см. формулу $\left.(11)\right)$, а именно $\sim[(k+1)(N+1)]^{-3}$. Возьмем в качестве примера очень короткую цепочку $(N=2)$, а в качестве аппроксимации - сумму всего четырех амплитуд $a_{0}+a_{1}+a_{2}+a_{3}$. Тогда в области $t<8$ ожидаемая погрешность будет $\sim 0.005$. На рис. 2 эта частичная сумма сравнивается с результатом, полученным численным интегрированием уравнения Шредингера (см. выражение (5)). Таким образом, мы видим, что даже для коротких решеток амплитуда $a(t)$ очень хорошо описывается суммой конечного числа парциальных амплитуд.

\section{3. РЕКУРСИЯ. ПЕРИОДИЧЕСКИЙ ВОЗВРАТ К НАЧАЛЬНОМУ СОСТОЯНИЮ}

Если решетка достаточно длинная, то следующие друг за другом парциальные амплитуды успевают затухнуть на соответствующем временно́м интервале $[t, t+N]$. В этом случае парциальные амплитуды не интерферируют, и на соответствующем временно́м интервале они очень хорошо воспроизводят полную амплитуду (см. рис. 3). Максимумы возвращения, однако, постепенно убывают.

В коротких решетках парциальные амплитуды интерферируют и достижение максимума $a(t)$ носит нерегулярный характер. На рис. 4 для цепочки с $N=5$ показан график амплитуды $a(t)$. Численное исследование для разных значений параметров $C, E$ и $N$ показало, что наибольшее значение амплитуды возврата составляет примерно 0.972 при времени $t=42$ для $N=10, E=0, C^{2}=0.4$. 


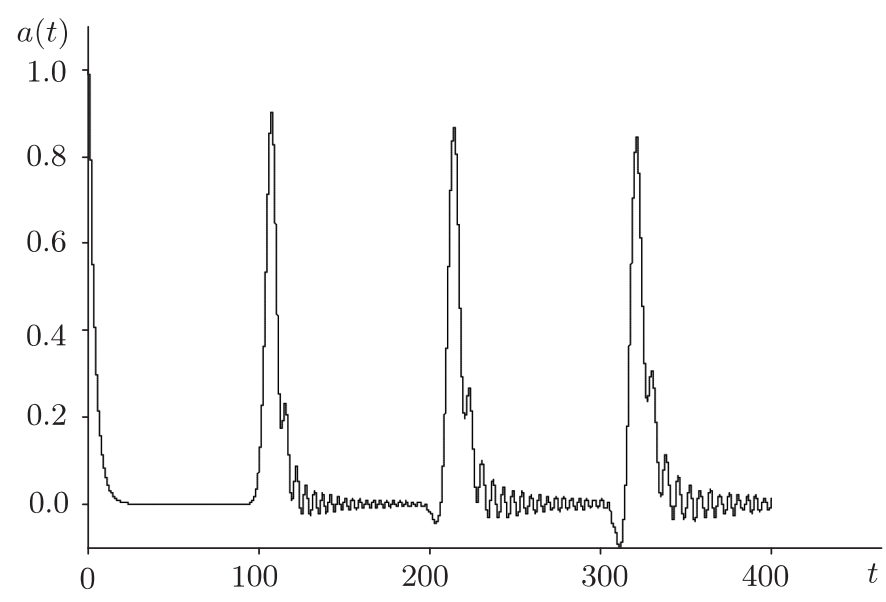

Рис. 3. Зависимость амплитуды от времени. Отдельно взятые парциальные амплитуды $a_{0}, a_{1}, a_{2}, a_{3}$ практически совпадают с полной амплитудой. Точные численные значения не показаны, так как они прекрасно совпадают с аналитическими результатами (среднеквадратическая ошибка $\lesssim 10^{-4}$ ). Значения параметров: $N=100, E=0, C^{2}=0.25$.

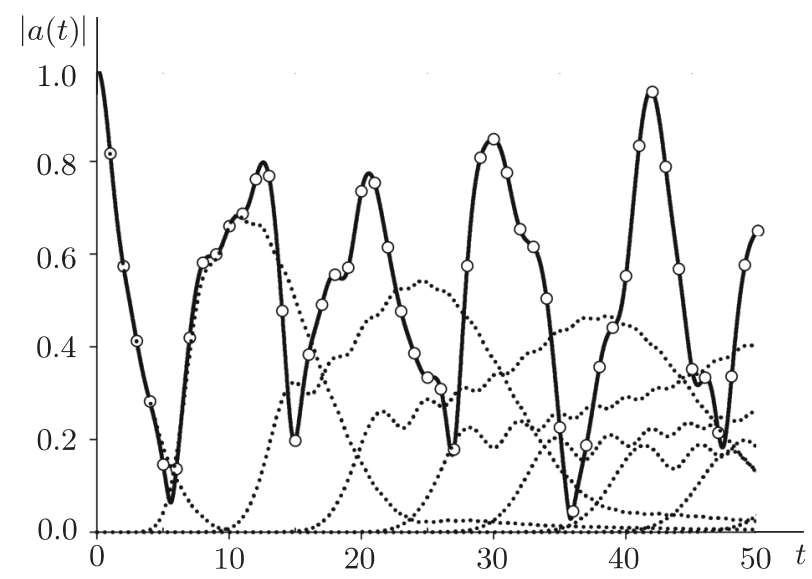

Рис. 4. График $a(t)$ для цепочки с $N=5$. Сплошная линия - сумма парциальных амплитуд $\left|a_{0}+a_{1}+\cdots+a_{8}\right|$. Практически она совпадает с амплитудой $a(t)$. Пунктиром показаны парциальные амплитуды $a_{0}, a_{1}, \ldots, a_{8}$. Максимальное значение амплитуды примерно равно 0.95 (при $t=42$ ). Видно, что основной вклад в этот максимум дают следующие парциальные амплитуды: $a_{2}, a_{3}, a_{4}, a_{5}, a_{6}$. Кружки - численные значения (среднеквадратичная ошибка $\left.\lesssim 10^{-4}\right)$.

Для малых решеток степень "возвращаемости" системы в начальное состояние для всех значений параметров $C, E, N$ исследовать довольно трудно из-за интерференции падающего и отраженного импульсов. Но мы можем рассмотреть первое 


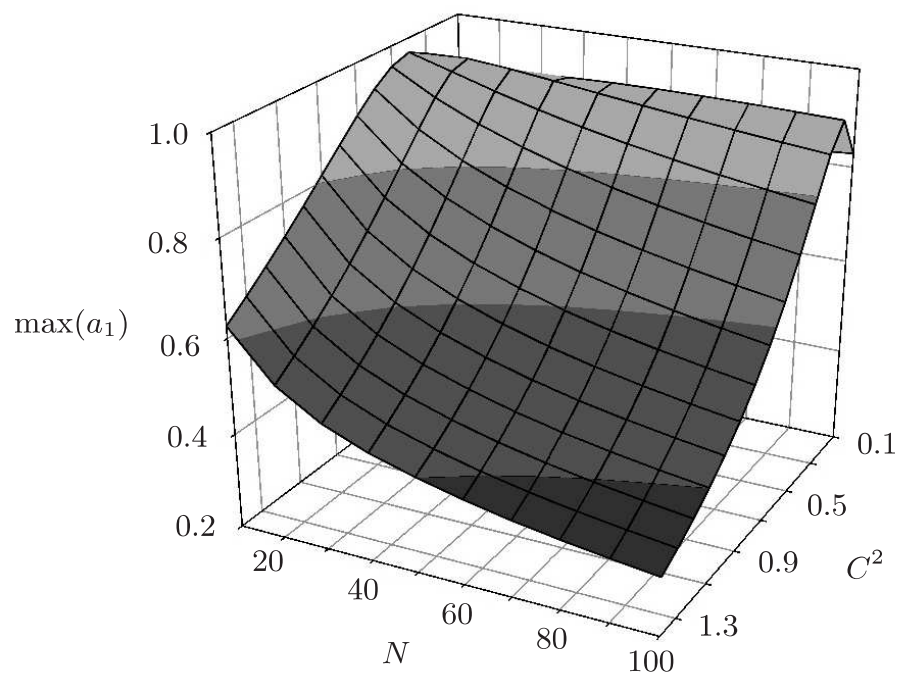

Рис. 5. Максимум амплитуды первого возвращения для случая $E=0$ и разных значений $C$ и $N$.

возвращение (максимум парциальной амплитуды $a_{1}$ ), считая цепочку достаточно длинной для того, чтобы амплитуду $a_{0}$ можно было считать “выгоревшей".

Формула для $a_{1}(t)$ (см. выражение $(23)$ ) с помощью тригонометрической замены переменной приобретает вид

$$
a_{1}(t)=\frac{4 C^{2}}{\pi} \int_{0}^{\pi} \sin ^{2} x e^{2 i t \cos x} \operatorname{Re}\left\{\frac{e^{2 i(N+1) x}}{\left[2 \cos x+E-C^{2} e^{i x}\right]^{2}}\right\} d x .
$$

По этой формуле вычислялся максимум амплитуды $a_{1}(t)$. На рис. 5 показан максимум $a_{1}(t)$ для случая $E=0$ (амплитуда в этом случае вещественна). Из рис. 5 видно, что при $C^{2} \approx 0.2$ "вернувшаяся" амплитуда примерно равна 0.9 и практически не зависит от длины решетки $(10<N<100)$. Отличие максимума парциальной амплитуды от максимума полной амплитуды в рассматриваемом временно́м интервале $(N<t<2 N)$ незначительно. Расхождение существенно только для самой короткой из рассматриваемых решеток $(N=10)$ и для самой маленькой константы связи $\left(C^{2}=0.1\right)$. При этих значениях параметров амплитуда $a_{0}$ просто не успевает затухнуть. Расхождение составляет $\sim 15 \%$.

\section{4. ЗАХВАТ ВОЛНОВОЙ ФУНКЦИИ АКЦЕПТОРОМ}

Рассмотренное выше явление многократного отражения волнового пакета и его концентрации на примесном узле вряд ли можно наблюдать экспериментально, поскольку волновая функция "вовне" себя никак не проявляет. В этом разделе мы рассмотрим задачу, достаточно близкую к экспериментам по переносу заряда, например в ДНК. В этих экспериментах регистрация переноса заряда осуществляется по фотофизическому и электрохимическому отклику акцептора на захваченный заряд, и интерес представляет то, какая доля полной волновой функции захватывается 


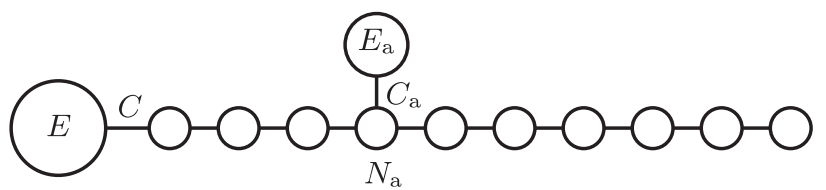

Рис. 6. Схема решетки с акцептором. К уже рассмотренной выше решетке с примесным узлом на левом конце с параметрами $E$ и $C$ сбоку к узлу с но-

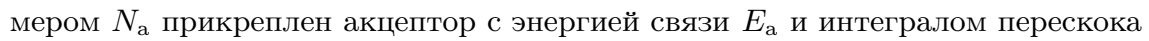
(связь с основной решеткой) $C_{\mathrm{a}}$.

акцептором в зависимости от параметров задачи. Эту величину можно сопоставить с квантовой эффективностью переноса заряда.

Рассмотрим цепочку, в которую введен дополнительный узел (акцептор), связанный с каким-либо из узлов решетки (см. рис. 6). Номер этого узла обозначим через $N_{\mathrm{a}}$. Акцептор имеет энергию $E_{\text {a }}$, константа связи с узлом $N_{\text {a }}$ равна $C_{\text {a }}$. Амплитуду волновой функции на акцепторе обозначим через $b_{\mathrm{a}}$. Сначала мы ограничимся случаем слабой связи акцептора с решеткой, т. е. будем полагать, что $C_{\mathrm{a}} \ll 1$. Это приближение позволяет провести аналитическое исследование задачи.

Система уравнений (5) изменится очевидным образом. Во-первых, добавится уравнение для амплитуды волновой функции на акцепторе:

$$
i \dot{b}_{\mathrm{a}}(t)=E_{\mathrm{a}} b_{\mathrm{a}}(t)+C_{\mathrm{a}} b_{N_{\mathrm{a}}},\left.\quad b_{\mathrm{a}}(t)\right|_{t=0}=0,
$$

а также изменится уравнение для узла с номером $N_{\mathrm{a}}$ :

$$
i \dot{b}_{N_{\mathrm{a}}}(t)=b_{N_{\mathrm{a}}-1}+b_{N_{\mathrm{a}}+1}+C_{\mathrm{a}} b_{\mathrm{a}} .
$$

Остальные уравнения системы (5) останутся без изменений.

Заметим, что известен способ введения поглощающего центра в решетку [2], который обеспечивает необратимый захват волновой функции на акцепторе. Если поглощающий центр находится на $n$-м узле, то уравнение для этого узла в системе уравнений (5) нужно записать в виде $i \dot{b}_{n}=b_{n-1}+b_{n+1}-i \hbar / \tau$, и $\tau$ есть характерное время захвата волновой функции.

Оставаясь в рамках первоначально сформулированной задачи, рассмотрим сначала результаты, полученные при численном моделировании в том случае, когда акцептор находится вблизи примесного узла. Оказывается, что модуль амплитуды волновой функции на акцепторе после достижения некоторого значения очень долго продолжает оставаться практически постоянным. Это явление проиллюстрировано на рис. 7 для цепочки с $N=100$, где время такое, что отраженный импульс не успевает дойти обратно до акцептора.

В приближении слабой связи $\left(C_{\mathrm{a}} \ll 1\right)$ можно вычислить зависимость амплитуды волновой функции на акцепторе от времени. Для этого заметим, что в случае слабой связи акцептор мало влияет на цепочку, поэтому ее можно считать невозмущенной и описывать ее уравнением (5). При этом амплитуду волновой функции на акцепторе будем вычислять согласно очевидному выражению, получающемуся из уравнения (25):

$$
b_{\mathrm{a}}(t)=-i C_{\mathrm{a}} e^{-i E_{\mathrm{a}} t} \int_{0}^{t} e^{i E_{\mathrm{a}} \tau} b_{N_{\mathrm{a}}} d \tau .
$$




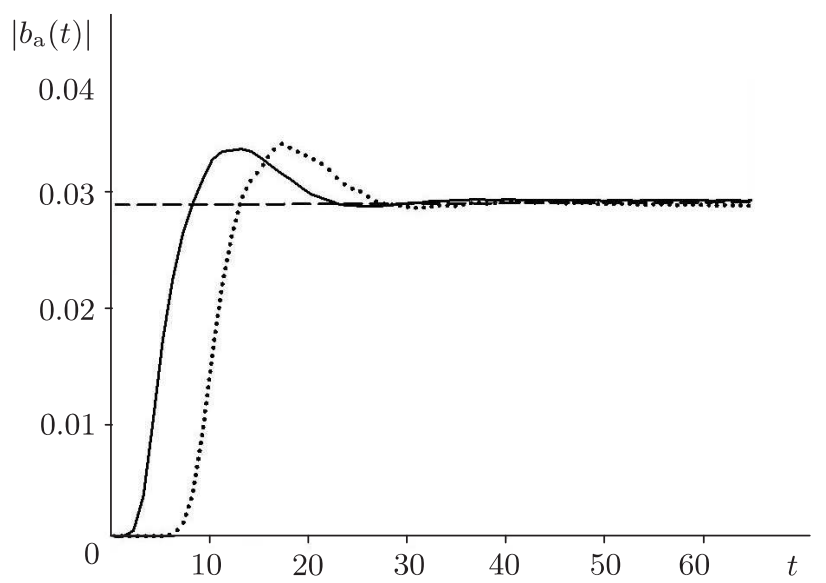

Рис. 7. Зависимость амплитуды волновой функции на акцепторе $\left|b_{\mathrm{a}}(t)\right|$ для двух значений положения акцептора на решетке: $N_{\text {a }}=5$ (сплошная линия) и $N_{\mathrm{a}}=15$ (пунктир). Штриховая линия - выражение (33). Значения параметров: $N=100, C=0.4, E=0.5, C_{\mathrm{a}}=0.02, E_{\mathrm{a}}=0.3$.

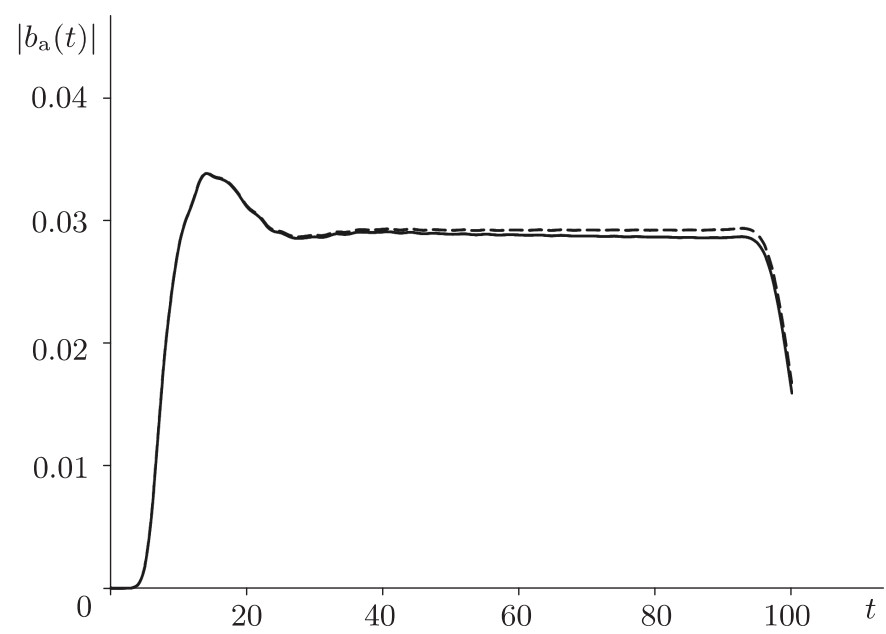

Рис. 8. Сравнение точного и приближенного решений для акцептора, связанного с десятым узлом $\left(N_{\mathrm{a}}=10\right)$. Сплошная линия - точное решение (учтено влияние акцептора на решетку), штриховая - невозмущенная решетка (акцептор не влияет на решетку). Значения параметров: $N=100, N_{\mathrm{a}}=10$, $C=0.4, E=0.5, C_{\mathrm{a}}=0.02, E_{\mathrm{a}}=0.3$.

На рис. 8 показано сравнение двух решений уравнения Шредингера: точного (выражение (25)) и приближенного (уравнения (5)). Видно, что эти решения отличаются очень мало, так что приближение невозмущенной решетки оказывается очень хорошим. 
Аналитически мы будем рассматривать случай, когда к интересующим нас временам произошло "выгорание" амплитуды $a(t)$ на примесном узле, а также и амплитуд на узлах, близких к акцептору, т. е. предполагается, что решетка достаточно длинная и время достаточно велико, так что импульс и "хвост" импульса ушли далеко от акцептора. Тогда можно считать, что до возвращения импульса цепочка ведет себя так, как будто она имеет бесконечную длину.

Как видно из выражения (27), нужно вычислить интеграл $\int_{0}^{t} e^{i E_{\mathrm{a}} \tau} b_{N_{\mathrm{a}}} d \tau$ (для модуля волновой функции фазовый множитель $e^{-i E_{\mathrm{a}} t}$ несуществен). Рассмотрим предельное значение амплитуды на акцепторе в пределе $t \rightarrow \infty$.

Для вычисления интегралов надо умножить систему уравнений (5) на $e^{i E t}$ и проинтегрировать от нуля до бесконечности. Введем обозначения:

$$
I_{0} \equiv \int_{0}^{\infty} e^{i E_{\mathrm{a}} \tau} a(\tau) d \tau, \quad I_{k} \equiv \int_{0}^{\infty} e^{i E_{\mathrm{a}} \tau} b_{k}(\tau) d \tau .
$$

Тогда для $I_{k}$ имеем систему рекуррентных соотношений:

$$
\begin{aligned}
C I_{1} & =-i+\left(E-E_{\mathrm{a}}\right) I_{0} \\
I_{2} & =E_{\mathrm{a}} I_{1}-C I_{0} \\
I_{3} & =E_{\mathrm{a}} I_{2}-I_{1} \\
I_{4} & =E_{\mathrm{a}} I_{3}-I_{2}
\end{aligned}
$$

В рассматриваемом приближении амплитуда на примесном узле $a(t)$ есть $a_{0}(t)$, а интеграл $I_{0}$ есть преобразование Лапласа $a_{0}\left(p=i E_{\mathrm{a}}\right)$. В результате можно получить

$$
I_{0}=\left[i\left(E-E_{\mathrm{a}}\right)+C^{2} e^{i \phi}\right]^{-1}, \quad \phi=\arcsin \frac{E_{\mathrm{a}}}{2} .
$$

Система уравнений (29) имеет следующее решение:

$$
I_{k}=C I_{0}\left[-i e^{i \phi}\right]^{k} .
$$

Таким образом, предельные значения амплитуд акцептора (с точностью до осциллирующего множителя $e^{-i E t}$ ) на разных узлах равны $-i C_{\mathrm{a}} I_{k}$ (см. уравнение $(26)$ ) и отличаются только фазовым множителем. При этом амплитуда на акцепторе равна

$$
b_{\mathrm{a}}(t) \approx-i C_{\mathrm{a}} C e^{-i E_{\mathrm{a}} t} I_{0} e^{\left[-i e^{i \phi}\right]^{k}} .
$$

Если исключить осциллирующие фазовые множители, несущественные для амплитуды волновой функции, то значение амплитуды равно

$$
b_{\mathrm{a}}(t) \approx \frac{C_{\mathrm{a}} C}{i\left(E-E_{\mathrm{a}}\right)+C^{2} e^{i \phi}}, \quad \phi=\arcsin \frac{E_{\mathrm{a}}}{2} .
$$

На рис. 7 видно, что предельное значение амплитуды очень хорошо совпадает с точным результатом численного расчета.

Из выражения (33) следует, что при фиксированных значениях интегралов перескока $C$ и $C_{\text {a }}$ значение $b_{\text {a }}$ будет максимальным при "резонансе" значений $E$ и $E_{\text {a }}$, т. е. при $E=E_{\mathrm{a}}$. И в этом случае $b_{\mathrm{a}}(t) \approx C_{\mathrm{a}} / C$. 


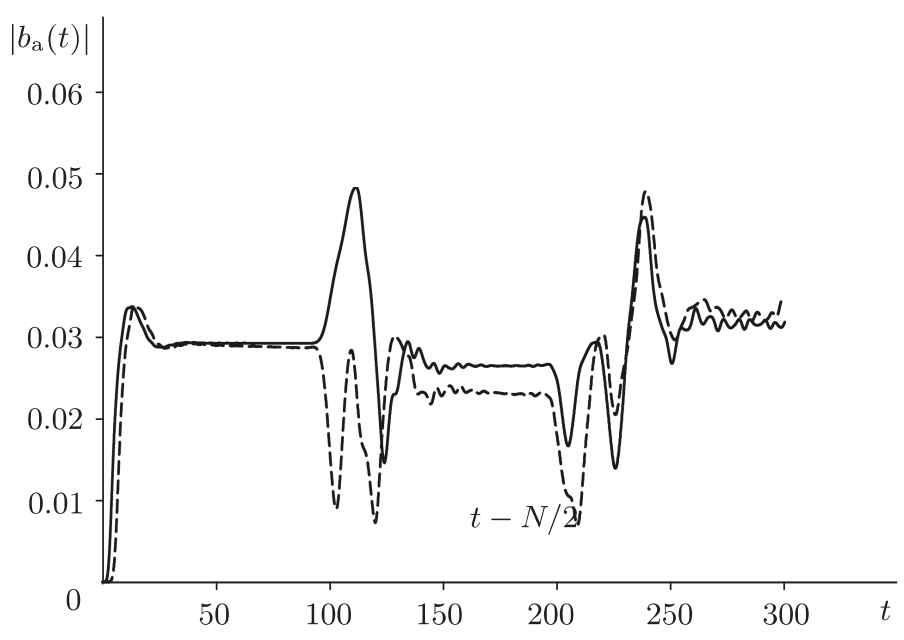

Рис. 9. Амплитуда волновой функции на акцепторе при $N_{\mathrm{a}}=5$ (сплошная линия) и $N_{\mathrm{a}}=10$ (штриховая линия), когда время таково, что происходит двукратное отражение волнового пакета. Значения параметров: $N=100$, $N_{\mathrm{a}}=10, C=0.4, E=0.5, C_{\mathrm{a}}=0.02, E_{\mathrm{a}}=0.3$.

Если времена таковы, что импульс отражается и возвращается обратно, то происходят изменения амплитуд в моменты прохождения фронта импульса мимо акцептора, и эти изменения носят нерегулярный характер, зависящий от положения акцептора (см. рис. 9).

Выше рассмотрены случаи, когда связь акцептора с основной цепочкой мала и амплитуда волновой функции на акцепторе невелика. Однако с практической точки зрения для эффективного переноса заряда необходимо, чтобы его вероятность была достаточно большой. Очевидно, что для этого связь акцептора с основной цепочкой должна увеличиться, т. е. параметр $C_{\text {a }}$ должен быть больше. Некоторое влияние на долю захваченной акцептором волновой функции оказывает и параметр $E_{\mathrm{a}}$.

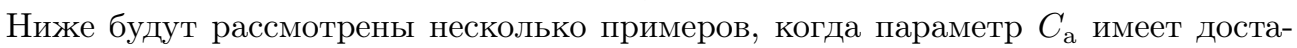
точно большое значение. На рис. 10 показана зависимость от времени амплитуды волновой функции на акцепторе, когда интеграл перескока $C_{\mathrm{a}}=0.1$. В этом случае амплитуда $\left|b_{\mathrm{a}}\right|$ становится заметно больше и достигает значений $\left|b_{\mathrm{a}}\right| \lesssim 0.2$.

В случае полного "резонанса" (когда $E=E_{\text {a и }} C=C_{\text {a) }}$ ) амплитуда $\left|b_{\mathrm{a}}\right|$ становится еще больше, и этот случай показан на рис. 11.

Из рис. 10, 11 следует, что заселенность акцептора может сильно изменяться при изменении параметров задачи. Видно, что когда амплитуда волновой функции на акцепторе невелика (рис. 10), то время жизни достаточно большое. И напротив, когда эта амплитуда велика, то время жизни мало (рис. 11). Это естественно, поскольку чем больше связь акцептора с решеткой, тем больше амплитуда захваченной волновой функции и тем меньше время жизни.

И, наконец, рассмотрим случай, когда акцептор расположен на конце решетки (рис. 12). Видно, что амплитуда волновой функции на акцепторе достаточно велика $\left(\left|b_{\mathrm{a}}\right| \approx 0.35\right)$ и, что очень важно, не зависит от длины решетки. Более того, 


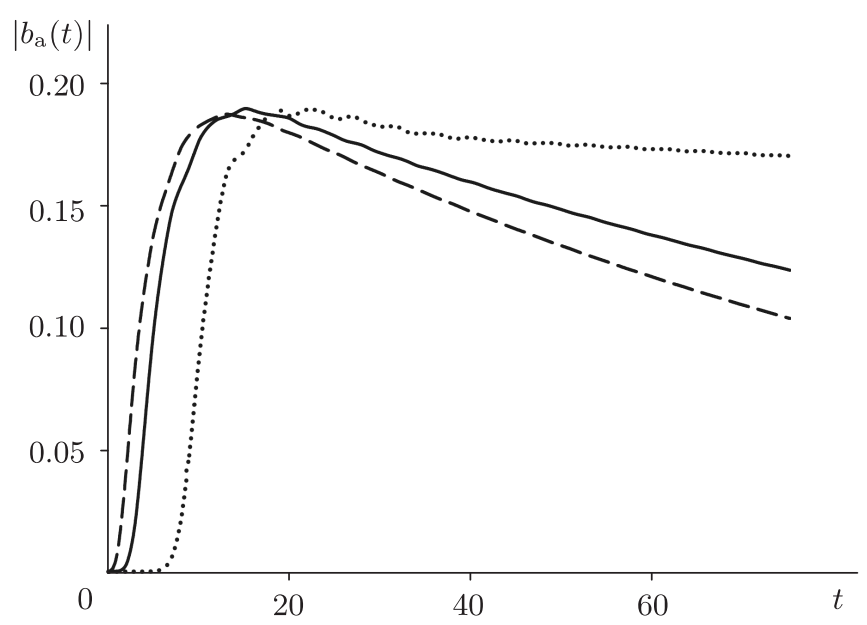

Рис. 10. Зависимость амплитуды волновой функции на акцепторе от времени для нескольких положений акцептора: $N_{\mathrm{a}}=2$ (штриховая линия), $N_{\mathrm{a}}=5$ (сплошная линия) и $N_{\text {a }}=15$ (пунктир). Значения параметров: $N=100$, $C=0.5, E=0.5, C_{\text {a }}=0.1, E_{\mathrm{a}}=0.5$ (согласно выражению (33) значение $\left.b_{\mathrm{a}}=0.2\right)$.

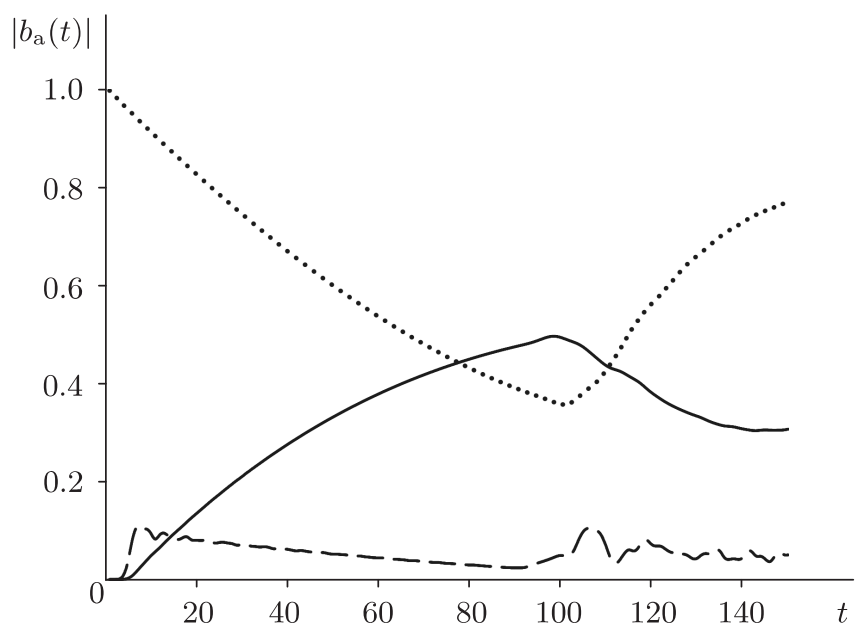

Рис. 11. Зависимость амплитуды волновой функции от времени на акцептоpe $\left|b_{\mathrm{a}}(t)\right|$ (сплошная линия), на примесном узле $|a(t)|$ на левом конце решетки (пунктир) и на 10-м узле, к которому присоединен акцептор $\left|b_{10}(t)\right|$ (штриховая линия). Значения параметров: $N=100, C=0.1, E=0.5, C_{\text {a }}=0.1$, $E_{\mathrm{a}}=0.5$. 


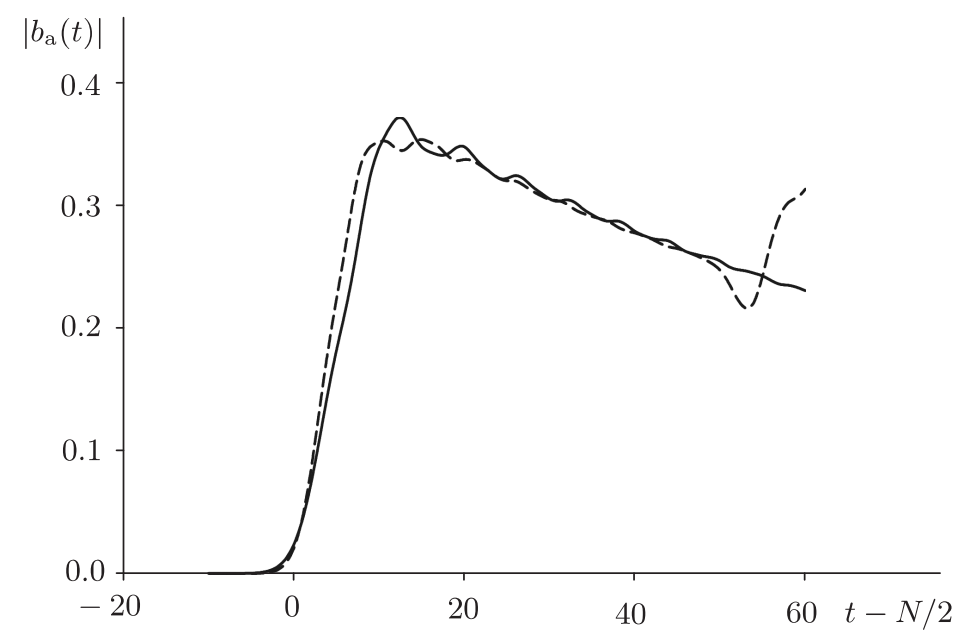

Рис. 12. Зависимость амплитуды волновой функции от времени на акцепторе, расположенном на конце решетки длиной $N=100$ (сплошная линия) и $N=50$ (штриховая). Значения параметров: $C=0.5, E=0.5, C_{\text {a }}=0.1$, $E_{\mathrm{a}}=0.5$. Для сравнения результатов начало отсчета времени сдвинуто назад для каждой кривой на величину $N / 2$ - время достижения импульсами конца цепочки.

временны́е закономерности уменьшения амплитуд почти одинаковы. Этот результат очень хорошо согласуется с экспериментами по переносу заряда в синтетических ДНК и полипептидах, где вероятность переноса заряда практически не зависит от расстояния.

В заключение этого раздела следует сделать некоторые оценки для характерных времен рассматриваемой задачи. Это нужно, для того чтобы понять, как долго волновая функция остается захваченной акцептором и хватит ли этого времени для реализации необходимого отклика (фотофизического или электрохимического) для регистрации заряда. Из анализа динамических и электронных свойств ДНК в аналогичной модели ДНК [3], [4] следует, что характерное (колебательное) динамическое время $[t]_{\mathrm{d}} \approx 1.7 \cdot 10^{-13} \mathrm{c}$, а характерное электронное время, которое и является единицей времени в настоящей работе, почти на два порядка меньше: $[t]_{\mathrm{e}} \approx 2.2 \cdot 10^{-15}$ с [5], [6]. Выше было показано, что заряд на акцепторе может удерживаться в течение нескольких десятков единиц времени, что может составлять несколько пс. И этого времени во многих случаях достаточно для возможности регистрации заряда.

\section{5. ЗАКЛЮЧЕНИЕ}

В статье [1] и в настоящей работе мы подробно исследовали распространение возбуждения и его отражения в квантовой системе, состоящей из однородной решетки и примесного центра. В настоящей работе рассмотрено также многократное отражение возбуждения от концов решетки и различные случаи захвата волновой функции акцептором. 
Исследован довольно неожиданный результат, заключающийся в том, что первоначально локализованная волновая функция начинает двигаться, образовывая хорошо локализованный волновой пакет. После отражения первоначально расплывающийся волновой пакет вновь концентрируется на примесном центре, и этот процесс повторяется много раз.

Для описания многократных отражений волнового пакета полезным приемом оказалось представление полной амплитуды волновой функции на примесном центре $a(t)$ в виде суммы парциальных амплитуд $a_{k}(t)$.

Поведение амплитуды $a(t)$ очень хорошо описывается аналитическими выражениями вплоть до десятка отражений, и на этих временах решение регулярно и не переходит в хаотический режим.

Полученные результаты при исследовании захвата волновой функции акцептором способны объяснить недавние экспериментальные результаты по баллистическому распространению заряда по синтетическим фрагментам ДНК.

ПРИЛОЖКНИЕ

Решение исходного уравнения (3) для лаплас-образа $a(p)$ имеет вид

$$
a(p)=\frac{1}{p+i E+B^{N}(p)} .
$$

Из представления Пуассона для ядра $B^{N}(p)($ см. выражения $(8),(17),(20))$, где было введено обозначение

$$
b(p) \equiv\left(i \widetilde{B}_{0}\right)^{2 k(N+1)},
$$

получаем

$$
B^{N}(p)=C^{2} B_{0}+C^{2} \sqrt{p^{2}+4} \frac{b}{1-b},
$$

поскольку $\widetilde{B}_{m}$ образуют геометрическую прогрессию.

Окончательное выражение для полной амплитуды имеет вид

$$
a(p)=a_{0} \frac{1-b}{1+b\left(C^{2} a_{0} \sqrt{p^{2}+4}-1\right)} .
$$

Разложение в ряд по $b$ дает лаплас-образы парциальных амплитуд, так что можно было не строить систему рекуррентных соотношений для парциальных амплитуд. Но такой способ действий годится только для данной задачи, в которой удается провести соответствующее суммирование и написать компактное выражение для ядра $B^{N}$. Предложенный же нами в статье способ разложения по парциальным амплитудам с помощью рекуррентных соотношений может быть применен и в других задачах.

Было численно проверено, что формула (П.1) действительно дает правильные ответы.

Обратное преобразование Лапласа дает искомое выражение для $a(t)$ :

$$
a(t)=\frac{1}{2 \pi} \int_{-\infty}^{\infty} a(p) d \omega, \quad p=\Delta+i \omega, \quad \Delta>0 .
$$


Отметим одно любопытное обстоятельство. Если “обернуть" интеграл (П.2) вокруг разреза $[-2 i, 2 i]$, то результат (разность интегралов по двум сторонам разреза) оказывается нулевым. Это было выяснено численно. Отсюда следует вывод, что в комплексной плоскости функция $a(p)$ имеет полюсы, и амплитуда $a(t)$ получается как сумма вычетов в этих полюсах. Для выяснения природы такого представления амплитуды требуется дополнительное исследование.

\section{Список литературы}

[1] В. Н. Лихачев, Т. Ю. Астахова, Г. А. Виноградов, ТМФ, 175:2 (2013), 279-299.

[2] Yu. A. Berlin, F. C. Grozema, L. D. A. Siebbeles, M. A. Ratner, J. Phys. Chem. C, 112:29 (2008), 10988-11000.

[3] E. M. Conwell, S. V. Rakhmanova, Proc. Natl. Acad. Sci. USA, 97:9 (2000), 4556-4560.

[4] E. M. Conwell, D. M. Basko, Synt. Met., 137:1-3 (2003), 1381-1383.

[5] Т. Ю. Астахова, В.Н.Лихачев, Г. А. Виноградов, Усn. хим., 81:11 (2012), 994-1010.

[6] Т. Ю. Астахова, В. Н. Лихачев, Г. А. Виноградов, Хим. физ., 31:12 (2012), 1-11.

Поступила в редакцию 26.10.2012, после доработки 27.11.2012 